\title{
Design of surface-modified electrodes for the electrochemical adsorption of Platinum-based anticancer drugs
}

\author{
Adam Kolodziej ja,b, Cécile S. Le Duffa, Julián Bergueiroc ${ }^{c}$, Marc Walkerd, Alexander Keelere, \\ Andrea E. Russelle, Paco Fernández-Trillo ${ }^{* a, b}$, \\ Paramaconi Rodriguez ${ }^{* a, b}$
}

\author{
a School of Chemistry, University of Birmingham, B15 2TT, Edgbaston, UK \\ ${ }^{\mathrm{b}}$ Birmingham Centre for Strategic Elements \& Critical Materials, University of Birmingham, B15 2TT \\ Edgbaston, UK. \\ c Freire Universität Berlin, Institute of Chemistry and Biochemistry, Takustr.3, 14195 Berlin, \\ Germany. \\ ${ }^{\mathrm{d}}$ Department of Physics, University of Warwick, CV4 7AL, Coventry, UK. \\ e School of Chemistry, University of Southampton, Highfield, Southampton, UK.
}

*Corresponding authors: f.fernandez-trillo@bham.ac.uk, p.b.rodriguez@bham.ac.uk

\begin{abstract}
The design of a hemofiltration method that minimizes the side effects produced by accumulation of Pt-based drugs such as cisplatin represents an important technology for cancer treatment. This work establishes the foundation for the development of an efficient platform for the electrochemical hemofiltration and simultaneous sensing of anti-tumour drugs, such as cisplatin and carboplatin, from physiological medium. The electrochemical filtration system is based on a gold surface-modified electrode using tris(carboxyethyl)phosphine (TCEP) ligands as scavengers of the Pt-based drugs complexes. The platinum-phosphine interaction is shown to be more time efficient, as compared to naturally occurring formation of a glutathione-platinum adduct and displayed a highly cooperative nature of binding. Altogether, we demonstrate that the presence of ligands capable of binding to Pt results in a differential spectroscopic and electrochemical response, and the potential to absorb and monitor the concentration of Pt salts in complex aqueous media.
\end{abstract}




\section{Introduction}

Since its discovery, cisplatin and its analogues have become important drugs in the treatment of several solid tumours. ${ }^{1-4}$ Even though the use of Pt-based drugs in chemotherapy has brought cure rates above $90 \%$, patients who undergo cisplatin-based chemotherapy are affected by several sideeffects. These side-effects are caused by the poor selectivity of the drugs for cancer tissues and the accumulation of Pt-based drugs in non-cancer tissues. ${ }^{5}$ In particular, a high systemic accumulation of platinum ${ }^{6}$ results in nephrotoxicity, gastrointestinal toxicity or neuropathy. ${ }^{7,8}$ In light of these side effects, the maximum dose of cisplatin to be administered has been reduced and consequently the chance of complete apoptosis of cancerous cells and full cancer remission have also been reduced. To overcome these disadvantages, number of methods to neutralise and remove cisplatin from blood have been investigated. For example, Florea et al. found that sodium thiosulfate can be used as a chelating agent to neutralize cisplatin in blood. However, thiosulfate also decreases the anticancer properties of the drug. ${ }^{8}$ An alternative approach, which has had positive results to a certain extent, is the filtration of Pt-based drugs using localized extracorporeal circuits or drug perfusion. These methods are advantageous as they enable a localized increase of drug levels in tumour tissues, up to ten-times higher than with systemic treatments, while minimizing at the same time long-term toxicities and side effects. Unfortunately, the haemoperfusion and hemofiltration methods reported so far are non-selective and suffer from low extraction efficiencies. ${ }^{9-13}$ Moreover, they do not provide a way of monitoring drug levels in blood.

Here, we report the use of surface-modified electrodes and electrochemical methods as a platform for the adsorption and monitoring of Pt-based drugs in physiological medium. The design of the reported surface-modified electrodes was underpinned by the identification of ligands capable of binding to platinum ions in complex aqueous media. Tris(carboxyethyl)phosphine (TCEP), the ligand with the highest affinity for Pt, was immobilized on the gold electrodes via coupling onto a selfassembled layer of cysteamine. The performance of the resulting surface-modified electrodes was assessed using cyclic voltammetry, impedance spectroscopy, X-ray photoelectron spectroscopy (XPS) 
and contact angle. Altogether, we demonstrate that the presence of ligands capable of binding to Pt results in a differential spectroscopic and electrochemical response, and the potential to absorb and monitor the concentration of Pt salts in complex aqueous media.

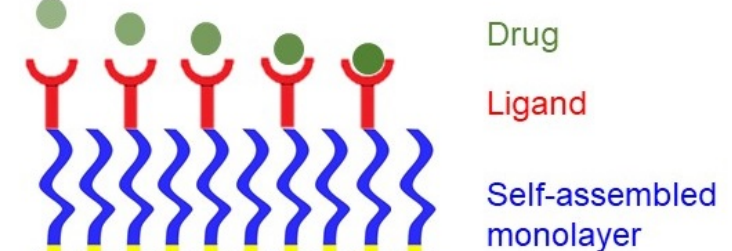
monolayer

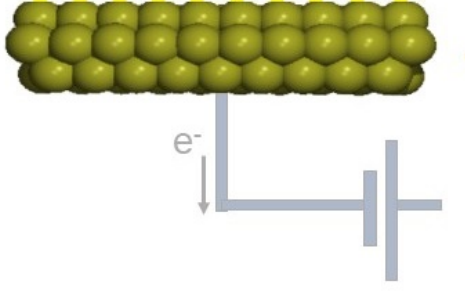

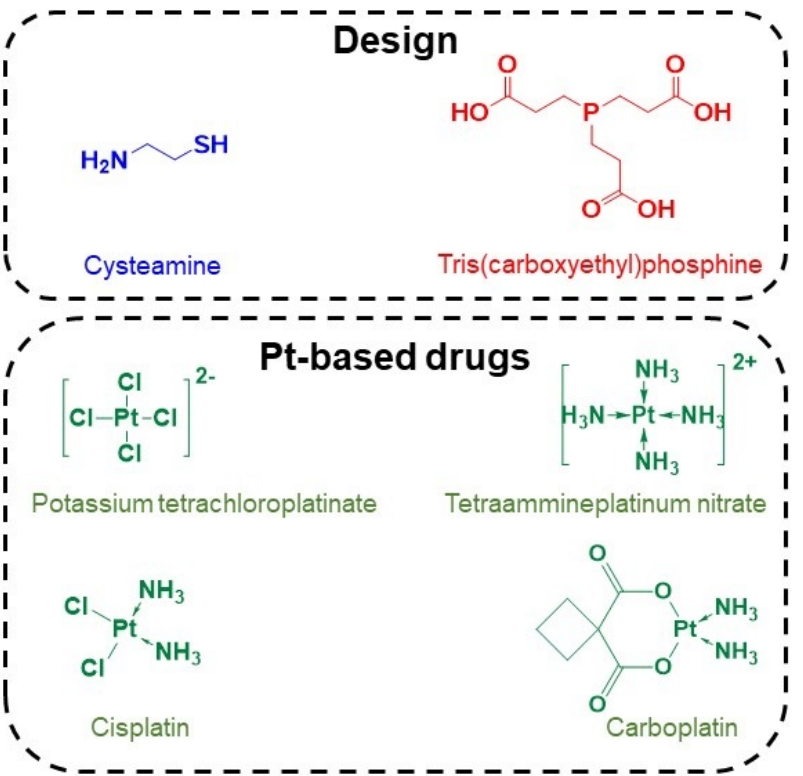

Figure 1: Schematic representation of the surface design of the working electrode for the adsorption and monitoring of Pt-based drugs along with molecules used to build the electrode and a scope of Pt-based compounds used throughout the study.

\section{Results and Discussion}

In nature, Pt-based drugs form stable adducts with the nitrogen in position $\mathrm{N}-7$ of guanine in nucleic acids. ${ }^{14}$ Additionally, glutathione (GSH) is known to scavenge the administered drug, due to the high affinity of thiols for Pt centres. ${ }^{15}$ To evaluate whether either of these two molecules had the potential to bind quickly to Pt-based drugs in complex aqueous media, they were mixed with 1 equivalent of cisplatin in a phosphate buffer saline solution, and the binding monitored via UV-Vis spectroscopy. The complexation of Pt in this physiological media is not trivial due to the affinity and competitive reaction with phosphate anions to metal centres. ${ }^{16,17}$ While no changes could be observed when guanine was used as the ligand (Fig. SI1), the interaction between cisplatin and GSH resulted in the appearance of a Pt-ligand band at $\sim 295 \mathrm{~nm}$ (Fig. SI2A). However, this band kept 
increasing even after $8 \mathrm{~h}$ of incubation, suggesting Pt-S binding for GSH was a slow process and that saturation was not achieved. In search for a suitable chelating agent for Pt-based drugs, we evaluated the affinity of other thiol-containing molecules searching for a faster kinetics of chelation of the Pt complex. To this end commercially available DL- thioctic acid (TOA) and 1,4-dithiothreitol (DTT) were investigated because both molecules have two thiols that could result in a higher affinity for the Pt centre in cisplatin (Fig. SI2B-C). The appearance of a ligand-to-metal band could be clearly observed after incubation of cisplatin with 1 equiv. of these thiol-containing molecules but, as before, no saturation could be observed even after $8 \mathrm{~h}$ of incubation.

Since Pt is a class (b) metal ${ }^{18}$ and preferentially binds to "heavier" atoms, we investigated then the ability of phosphorous containing molecules to scavenge Pt-based drugs from aqueous solution. To this end, 1 equiv. of water-soluble phosphine TCEP was incubated in the presence of cisplatin and the resulting UV-Vis spectrum recorded as a function of time (Fig. SI2D). As before, a ligand to Pt band could be observed in this case at $\sim 305 \mathrm{~nm}$. More importantly, saturation of this band could be observed even after only $1 \mathrm{~h}$ of incubation, suggesting that Pt-P binding was much faster than for any of the other molecules investigated. One of the parameters that define a good ligand for the capture of the Pt- drugs is the reaction time. A fast reaction between the Pt-based drug and the electrode, will avoid the distribution and recirculation of the drug in the body through the blood, thus reducing the side effects. In addition, will enable fast detection times.

To further investigate the binding of TCEP to Pt, samples were prepared again, and the kinetics of the binding now monitored using a quartz cuvette that allowed us to identify any bands resulting from Pt-P binding in the $210-300 \mathrm{~nm}$ window (Fig. 2A). An absorption band at $230 \mathrm{~nm}$ could now be observed that we attributed to the formation of the complex TCEP-cisplatin, in agreement to what has been reported in the literature. ${ }^{19}$ The intensity of this band quickly increased until it reached a plateau indicating that the reaction was completed within $1 \mathrm{~h}$ (Fig. 2B). Addition of 2 and 4 equiv. of TCEP had very little effect on the rate of the coordination but resulted in higher overall intensities, suggesting that more of the UV-responsive bonds could be formed under these conditions. A similar 
effect was observed for the reaction between TCEP and carboplatin, although reaction rates were now slower, as anticipated due to the presence of a bulky bidentate ligand in this Pt-based drug.
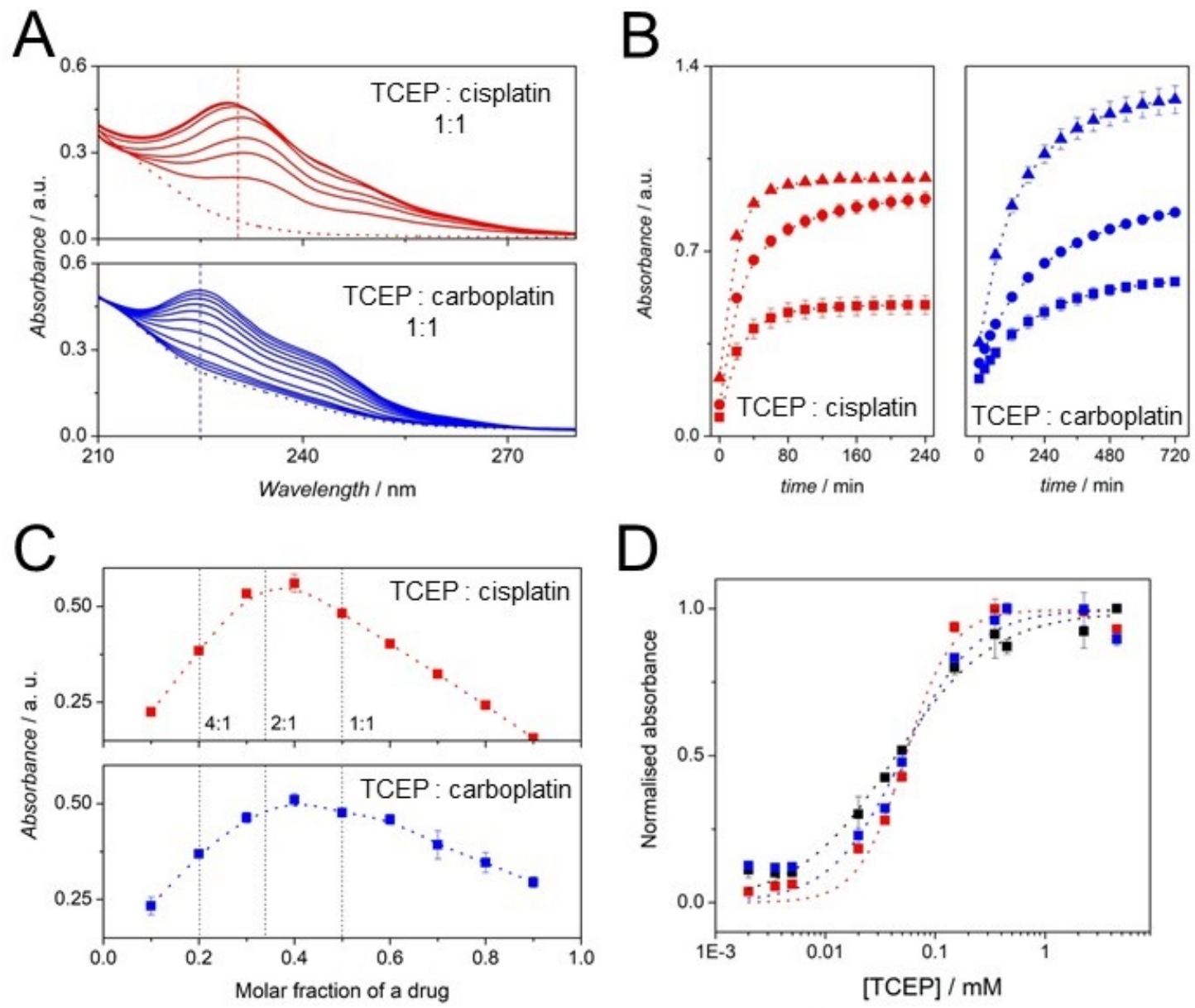

D

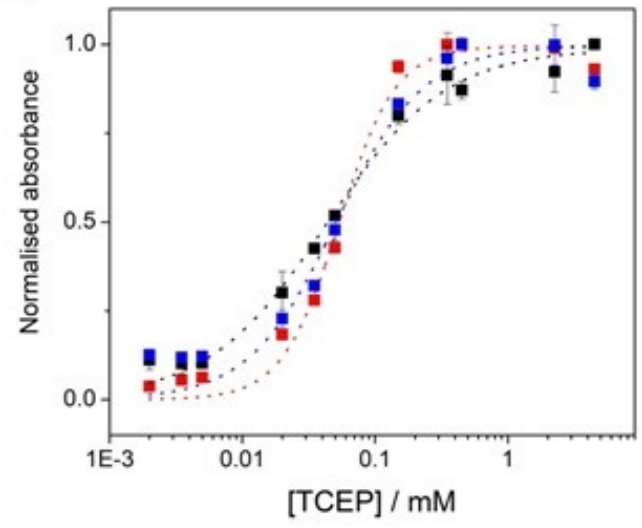

E

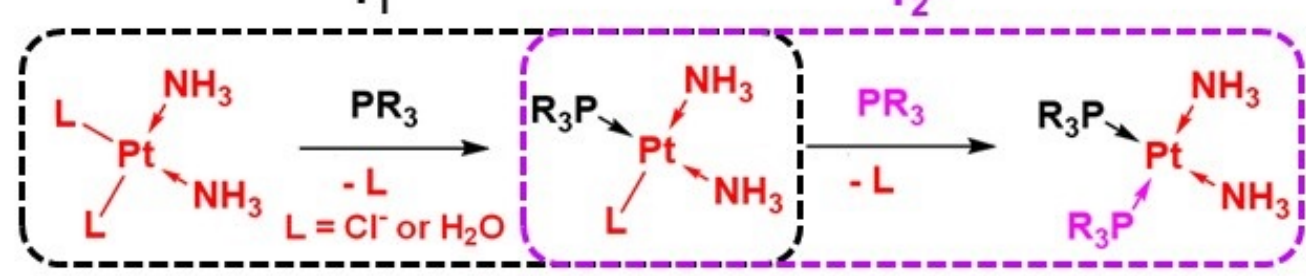

\section{Cooperative effect: $r_{1}<r_{2}$}

Figure 2: (A) UV-Visible spectroscopy monitoring of the reaction progress for the coordination of TCEP to cisplatin and carboplatin at 1:1 molar ratio. Dotted lines correspond to as mixed samples whereas solid lines indicate consecutive spectra. (B) Time-dependence intensity of the signal at $230 \mathrm{~nm}$ and $225 \mathrm{~nm}$ for the TCEP coordination to cisplatin and carboplatin, respectively at molar ratios of 1:1 ( $\square, \square), 2: 1(\bullet, \bullet), 4: 1(\Delta, \Delta)$. (C) Job plots for the complexation process between TCEP and cisplatin/carboplatin suggesting a 2:1 binding ratio. (D) Hill plots for binding between 
TCEP and potassium tetrachloroplatinate ( $\square$ ), cisplatin ( $\square$ ), and carboplatin ( $\square$ ). It suggests a cooperative binding in the case of cisplatin $(n=2.04 \pm 0.35)$ and carboplatin $(n=1.33 \pm 0.29)$ as compared to a non-cooperative binding of $\mathrm{K}_{2} \mathrm{PtCl}_{4}(n=0.97 \pm 0.09)$ with a consistent value of dissociation constant. (E) A schematic representation of the ligand exchange on cisplatin substitution by two TCEP molecules with apparent cooperative effect.

Job plots revealed that the coordination between TCEP and both Pt-based drugs was mainly via a 2:1 binding ratio, with a clear maximum of absorbance observed when that stoichiometry was employed (Fig. 2C). While the presence of more labile ligands, as in the case of chlorides for potassium tetrachloroplatinate $\left(\mathrm{K}_{2} \mathrm{PtCl}_{4}\right)$, had a beneficial effect on the rate of the complexation (Fig. SI3A-B), it did not affect the stoichiometry of the binding ratio between TCEP and Pt (Fig. SI3C). Therefore, under the studied reaction conditions, each Pt centre could be only coordinated by a maximum of two TCEP molecules. Alternatively, replacing the first coordination sphere ligands for $\mathrm{NH}_{3}$, which are not good leaving groups, ${ }^{20}$ resulted in no apparent coordination of TCEP to Pt (Fig. SI4), suggesting that TCEP mainly coordinates to the Pt-centre by replacing chloride or water ligands. It should be noted that in aqueous media, Pt-based drugs undergo ligand substitution where chlorides can be substituted by water molecules. ${ }^{21}$ The binding of one molecule to the metal centre can induce changes to the electronic properties and configuration of the complex, resulting in different complexation kinetics for a second ligand. ${ }^{22}$ To investigate whether binding of a TCEP molecule to Pt could result in a positive cooperative effect, where the binding of a second ligand would be favoured, Pt-based drugs were incubated with increasing amounts of TCEP and the changes in absorbance monitored. Fitting the normalised increase in absorbance observed to a Hill model ${ }^{23}$ (Fig. 2D) indicated that the coordination of TCEP to cisplatin exhibited a cooperative effect, with a Hill constant $(n)$ of $2.04 \pm 0.35$. This value suggests that upon the coordination of a TCEP molecule to the Pt-centre, the coordination of a second molecule to the Pt-centre is facilitated. On the other hand, the Hill constant for carboplatin was $1.33 \pm 0.29$, indicating a lower level of cooperativity, possibly as 
a result of the poorer leaving group character of the large organic ligand as compared to chlorides. Interestingly, increasing the number of good leaving groups results in the counter-intuitive loss of cooperativity, as indicated by the Hill constant for $\mathrm{K}_{2} \mathrm{PtCl}_{4}=0.97 \pm 0.10$. We propose that the absence of cooperativity for the binding of TCEP and $\mathrm{K}_{2} \mathrm{PtCl}_{4}$ could be associated to the electronic screening or buffering across the remaining chloride ligands. In line with these observations, affinity constants were determined to be $0.0533 \pm 0.0047,0.0508 \pm 0.0090$ and $0.0431 \pm 0.0051$ for cisplatin, carboplatin and potassium tetrachloroplatinate, respectively.

Further insight into the coordination of cisplatin or potassium tetrachloroplatinate with TCEP was achieved by monitoring the complexation via ${ }^{31} \mathrm{P}$ NMR (Fig. SI5 and Fig. SI6). In addition to the 2:1 TCEP:Pt stoichiometry described above, complex scenarios for the coordination should be considered, including the formation of different isomers. In our case, incubation of the Pt-containing molecules with TCEP (Fig SI5 and SI6, B and C spectra) resulted in the formation of two distinctive sets of signals. Two singlets at approximately $12-13 \mathrm{ppm}$ were attributed to the cis- and trans-oriented di-substituted products assuming possible inversion of the configuration through a pentacoordinate state..$^{24,25} \mathrm{An}$ additional singlet at 7-8 ppm could be attributed to the second sphere coordination of a TCEP molecule. In the case of $\mathrm{K}_{2} \mathrm{PtCl}_{4}$ (Fig. SI6), another singlet at $\sim 6.5 \mathrm{ppm}$ was also observed. We attribute this signal to the mono-substituted product which should be present due to the lack of cooperativity observed for this Pt salt.

The nature of the second sphere coordination signal at 7-8 ppm was confirmed when $\mathrm{Pt}\left(\mathrm{NH}_{3}\right)_{4}\left(\mathrm{NO}_{3}\right)_{2}$ was used instead of the Pt-based drugs. As discussed before, this Pt salt does not undergo ligand displacement in the first sphere of coordination and only the signal at $~ 7 \mathrm{ppm}$ was observed (Fig. SI7). This second sphere coordination in $\mathrm{Pt}\left(\mathrm{NH}_{3}\right)_{4}{ }^{2+}$ salts had been previously described. ${ }^{26,27}$ Increasing the ratio of TCEP did not result in the appearance of any new signal, beyond the presence of uncoordinated TCEP at $\sim-16 \mathrm{ppm}$ (Fig. SI7). Finally, performing the experiments in the absence of $\mathrm{NaCl}$ facilitated the $\mathrm{Cl} / \mathrm{H}_{2} \mathrm{O}$-to-TCEP exchange and as a result no second sphere coordination band was 
observed for both $\mathrm{K}_{2} \mathrm{PtCl}_{4}$ and cisplatin (Fig. Sl8A-B). As expected, this was not the case for $\mathrm{Pt}\left(\mathrm{NH}_{3}\right)_{4}\left(\mathrm{NO}_{3}\right)_{2}$, for which only second sphere coordination could be observed in any of the buffers investigated (Fig SI8C).

Once it was determined that the TCEP was the most suitable ligand for the coordination of cisplatin or carboplatin and understanding of the nature of the Pt-P interaction was achieved, the surface of a gold electrode was functionalized to introduce this Pt scavenger. Figure $3 \mathrm{~A}$ shows the schematic representation of the step-by-step immobilization of TCEP. Cysteamine (CA) was selected as a linker based on the presence of a terminal amine group and the electrochemical stability of its Au-layers in physiological medium. ${ }^{28}$ Immobilization of cysteamine and TCEP was monitored by cyclic voltammetry of the surface-modified gold electrodes at different stages of the modification (Fig. 3B). The self-assembled monolayer of cysteamine on gold was stable in a broad potential region down to $0.1 \mathrm{~V}$ vs RHE with the signal below $0.1 \mathrm{~V}$ vs RHE associated to the reductive desorption of cysteamine.$^{28}$ Following modification with cysteamine, TCEP was immobilized on the cysteamine layer through EDC coupling. ${ }^{29,30}$ No significant changes in the voltammetry profiles were observed confirming that the step-by-step surface modification did not affect the stability of the layer in the potential window under investigation and only a small shift of $25 \mathrm{mV}$ in the desorption peak towards less positive potential was observed. This shift can be attributed to the larger size of the CA-TCEP adlayer which results in higher energy needed to desorb the adlayer from the gold surface as compared to the cysteamine layer. ${ }^{31}$ This shift was not observed when the Au-CA electrode was exposed to the solution of EDC, confirming the TCEP coupling (Fig. SI9). The step-by-step surface modification and integrity of the layer was also confirmed by electrochemical impedance spectroscopy. As shown in Fig. 3C, phase shift plots showed a clear difference between the pristine gold electrode and the surface-modified electrodes. In these plots, a phase angle (- $\phi)$ of $90^{\circ}$ in the frequency domain at $1 \mathrm{~Hz}$ to $1 \mathrm{kHz}$ indicates the presence of an ideal capacitor, while lower angles are related to current leakage on defect sites of the layer. ${ }^{32}$ In our case, the phase angles for the 
surface modified electrodes at $1 \mathrm{~Hz}$ were $\phi=78^{\circ}$ which indicated a pseudocapacitive behaviour. This response could be associated to the presence of some defects in the layer or to the formation of a less compact film that would allow the permeation of ions such as $\mathrm{K}^{+}, \mathrm{HPO}_{4}{ }^{2-}$, and $\mathrm{H}_{2} \mathrm{PO}_{4}{ }^{-}$through the layer. ${ }^{32,33}$ The presence of inhomogeneities in the layer or the presence of a less compact layer could also be the cause of the local minimum at $10 \mathrm{~Hz}$. While the formation of SAMs of long-chain alkane thiols gives compact layers due to Van der Waals interactions, the presence of functional groups in the CA-TCEP layer architecture could lead to the presence of less compact layers. At high frequencies $(>10 \mathrm{kHz})$, the total impedance is dominated by the solution resistance. Finally, evidence of the functionalization of the gold electrodes with TCEP was obtained using contact-angle measurements. As expected, the contact angle decreased upon functionalization with TCEP (Fig 3D), indicating that the surface had become more hydrophilic when compared to the pristine gold electrode.

A
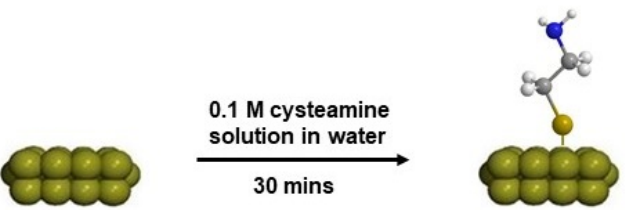

$0.02 \mathrm{M}$ TCEP solution in MES buffer $+0.08 \mathrm{M}$ EDC solution in MES buffer 30 mins

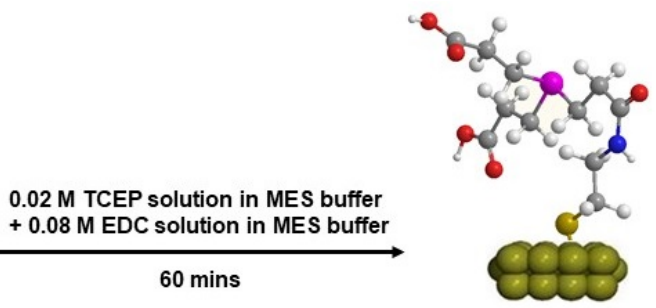

B

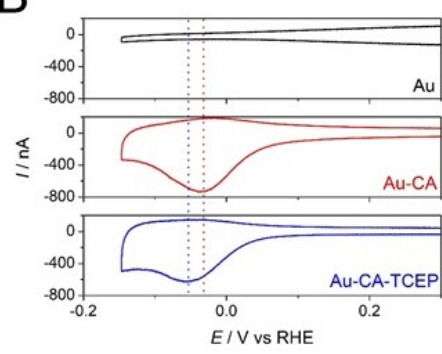

C

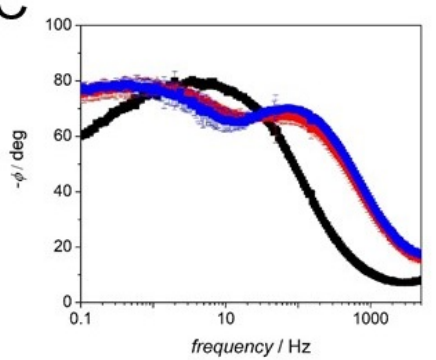

$\mathrm{D}$

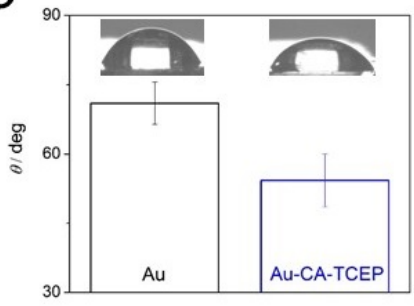

Figure 3: (A) Schematic representation of the strategy for the surface modification of the gold electrodes. (B) Voltammetric profiles of electrode at different stages of modification. Selfassembly of cysteamine $(-)$ results in the presence of reductive desorption peak at low potential value. This peak shifts by $30 \mathrm{mV}$ once TCEP is coupled to self-assembled cysteamine (-), (C) Bode plots of phase shift vs. frequency showing Au ( $\square)$, Au-CA ( $\square)$, Au-CA-TCEP ( $\square)$. Au-CA-TCEP shows a clear difference between unmodified and modified electrodes, but no change between Au-CA and 


\section{Au-CA-TCEP, suggesting that the integrity of the initial CA SAM is not affected by coupling of TCEP. (D) Contact angles for the wetting of Au and Au-CA-TCEP with deionised water.}

Once the stability of the TCEP-modified electrode was confirmed, the adsorption of Pt-based drugs and the electrochemical response were examined by cyclic voltammetry, electrochemical impedance spectroscopy, X-ray photoelectron spectroscopy and contact angle measurements. Upon adsorption of cisplatin on the TCEP-modified electrode, the oxidation state of the Pt was analysed by ex-situ XPS, where bands at $72 \mathrm{eV}$ and $75.3 \mathrm{eV}$ corresponding to the $\mathrm{Pt} 4 f_{7 / 2}$ and $\mathrm{Pt} 4 f_{5 / 2}$ components arising from $\mathrm{Pt}(\mathrm{II})$-Cl confirmed the presence of non-reduced cisplatin adsorbed on the TCEP-modified gold electrode (Fig 4A, bottom). On the other hand, cyclic voltammetry of the TCEP-modified electrode after exposure to cisplatin indicated that the capacitive current slightly increased in the region between 0.8 and $0.3 \mathrm{~V}$ but no obvious Faradic process was observed (Fig 4B). In the second cycle, no relevant changes in the voltammetric profile were observed. These results suggested that cisplatin was adsorbed on the TCEP-modified electrode but it was not reduced to $\mathrm{Pt}(0)$ in the given potential window. The adsorption without reduction to $\mathrm{Pt}(0)$ is an important parameter to consider for future applications as the formation of $\mathrm{Pt}(0)$ species might result in the electrochemical oxidation/reduction of other species present in the blood. To confirm this observation, this electrode was also analysed by XPS after cycling (Fig. 4A, top). In this case, the signal of Pt(II)-Cl slightly reduced, with only a small fraction ( $<15 \%)$ at $74 \mathrm{eV}$ and $71 \mathrm{eV}$ associated to reduced $\mathrm{Pt}(0)$. We believe reduction of the adsorbed cisplatin on the TCEP-modified electrode to $\mathrm{Pt}(0)$ would be achievable only at much lower potentials, potentials at which the reductive desorption of the self-assembled layer would also be observed (Fig. SI11). ${ }^{28}$ These results were in contrast with those obtained for the adsorption and electrochemical reduction of cisplatin on a bare Au electrode, where the reduction of cisplatin occurred at $0.48 \mathrm{~V}$ vs RHE (Fig. SI10). XPS analysis of this bare gold electrode after exposure to cisplatin confirmed that different behaviours were observed when depositing cisplatin on functionalised and unmodified gold electrodes with a significantly larger amount of reduced Pt being observed in the latter case (Fig SI12). 
However, this effect can be associated to the photoreduction of the platinum complex on gold induced by the X-rays, although sample exposure was minimized in an effort to negate these effects. ${ }^{34,35}$ The interaction of TCEP-modified electrodes with cisplatin was also monitored via EIS and the results obtained compared to those of the bare gold electrode. On the bare gold electrode, as the potential decreased the resistive response appeared at a lower frequency (Fig. SI13A, top) as the reduction of platinum took place on the surface of the gold electrode. On the other hand, the phase angle profiles for the TCEP-modified electrodes (Fig. SI13A, bottom) with cisplatin did not show any differences as the potential was stepped more negative suggesting that the binding on Au-CA-TCEP and the reduction of cisplatin did not take place on the pinholes of the layer where Au remains uncovered. Similar observations were made using $\mathrm{K}_{2} \mathrm{PtCl}_{4}$ as a source of Pt (Fig. SI13B). In order to gain more information on the interaction of the surfaces and the different Pt-based drugs electrochemical impedance spectroscopy measurements were performed. The Nyquist plot and the corresponding analysis can be found in Fig. SI14). Finally, contact angle measurements showed that upon adsorption of cisplatin on the CA-TCEP-modified electrode, its surface becomes more hydrophobic (Fig SI15). 

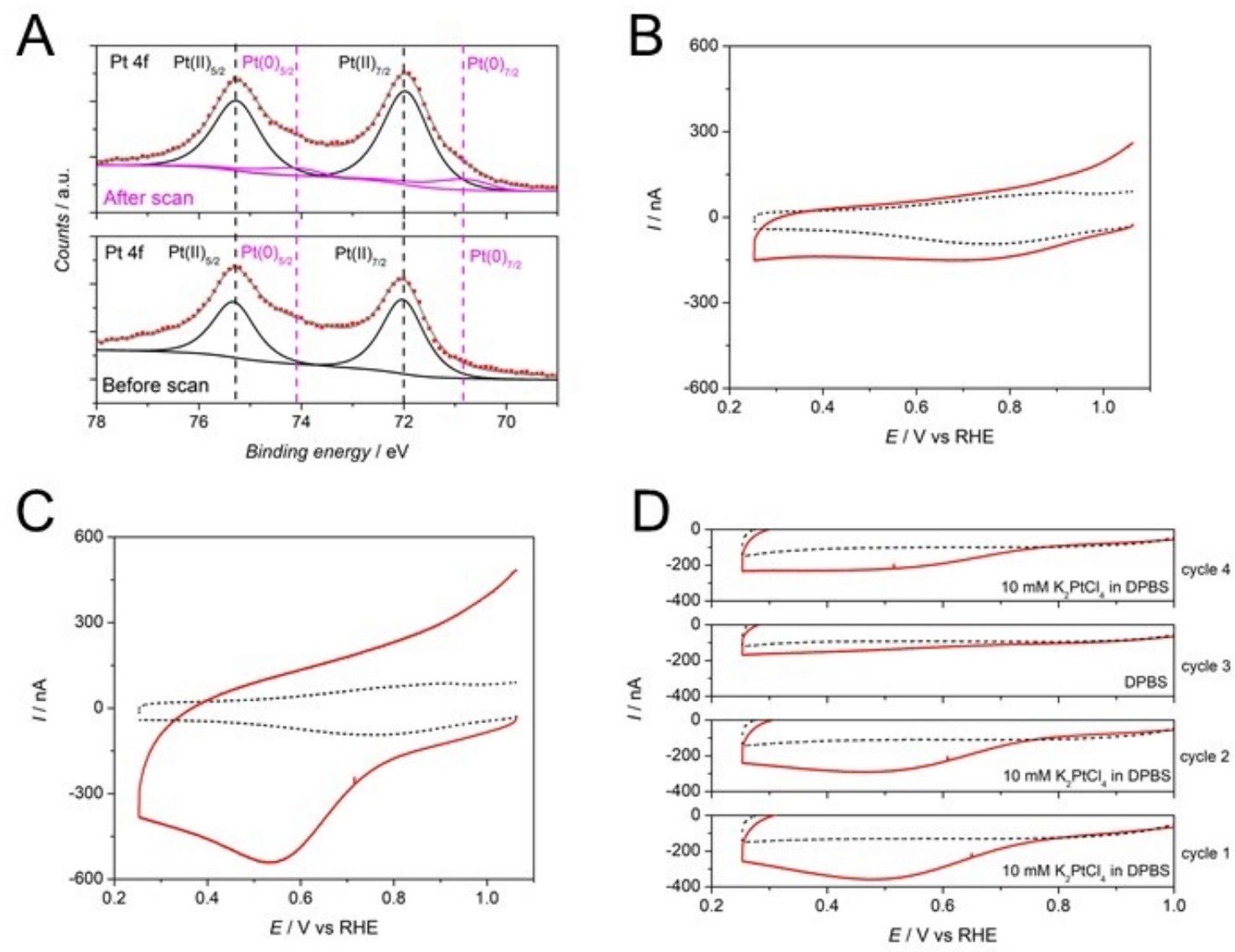

Figure 4: (A) XPS spectra of Au-CA-TCEP exposed to $1 \mathrm{mM}$ solution of cisplatin for $\mathbf{3 0}$ mins before and after electrochemical conditioning (a scan in the potential range as the one in the Fig. 4B). (-) full spectra and the corresponding fitting $(-),(-)$ and $(-)$ peak convolution of the $\mathrm{Pt}(\mathrm{II})$ and $\mathrm{Pt}(0)$ species, respectively. Vertical dashed lines $\left(\begin{array}{l}1 \\ 1\end{array}\right)$ and $\left(\begin{array}{l}1 \\ 1\end{array}\right)$ are shown for the sake of comparison. Voltammetric profiles of Au-CA-TCEP before and after exposure to (B) $1 \mathrm{mM}$ solution of cisplatin in DPBS for 30 mins, (C) $10 \mathrm{mM}$ solution of $\mathrm{K}_{2} \mathrm{PtCl}_{4}$ in DPBS for 15 mins. (-) correspond to experiments after exposure, whereas (‥) stand for blanks. (D) Adsorption experiment where AuCA-TCEP was immersed in $10 \mathrm{mM}$ solution of $\mathrm{K}_{2} \mathrm{PtCl}_{4}$ and electrochemically reduced multiple times (cycle 1, 2 and 4). A control experiment where the electrode was immersed in Pt-free DPBS (cycle 3) shows no reductive feature. $(-)$ corresponds to experiments after exposure, whereas $(\cdots)$ are blanks voltammograms.

The adsorption and electrochemical assessment were also performed on the CA-TCEP-modified electrode after adsorption of $\mathrm{PtCl}_{4}{ }^{2-}$. In contrast to the results obtained with cisplatin on CA-TCEP- 
modified electrodes, the voltammetric profile in Fig. $4 \mathrm{C}$ shows a reductive current contribution in the region between 0.7 and $0.3 \mathrm{~V}$ vs RHE corresponding to the reduction of $\mathrm{Pt}(\mathrm{II})$ to $\mathrm{Pt}(0)$. Interestingly, this signal was clearly different from that observed for the reduction of $\mathrm{PtCl}_{4}{ }^{2-}$ to $\mathrm{Pt}(0)$ on a bare gold electrode (Fig. SI16A). In order to gain further insight into the process taking place on the Au-CA-TCEP electrode, and discard the possibility that the reduction of $\mathrm{PtCl}_{4}{ }^{2-}$ to $\mathrm{Pt}(0)$ was occurring on the underlying gold electrode, experiments of consecutive cycles of adsorptions of $\mathrm{PtCl}_{4}{ }^{2-}$ and reduction experiments were performed. According to the nucleation and growth process, the formation of a nucleation centre - $\mathrm{Pt}(0)$ cluster - during the first cycle of $\mathrm{PtCl}_{4}{ }^{2-}$ absorption, will favour the growth of such clusters in the following adsorption/reduction cycles. As a result, the signal of the reduction of the adsorbed $\mathrm{PtCl}_{4}{ }^{2-}$ should appear at more positive potentials. ${ }^{36,37}$ On the other hand, if the $\mathrm{PtCl}_{4}{ }^{2-}$ is adsorbing on the available TCEP sites, the signal associated to the adsorption of $\mathrm{PtCl}_{4}{ }^{2-}$ should decrease as number of available sites decreases in each cycle. As can be seen in Fig. 5.4D, consecutive adsorption/reduction cycles of the $\mathrm{PtCl}_{4}{ }^{2-}$ lead to reduction signals appearing in all voltammograms (except a control experiment with DPBS only - cycle 3) with no changes to the onset potential for the reduction process being observed.. These results confirm that the growth of the nucleus was not taking place but that consecutive cycles lead to the adsorption of less $\mathrm{PtCl}_{4}^{2-}$ on TCEP sites. More importantly, the presence of this reductive current provides a means to monitor the adsorption of $\mathrm{PtCl}_{4}{ }^{2-}$ and this ability to monitor the adsorption process makes the concept of electrochemical hemofiltration even more versatile, ultimately providing a way to follow the amount of a drug in the blood stream. Our preliminary results have shown that adsorption can be qualitatively measured by the integration of charge involved in the irreversible electrochemical reduction of $\mathrm{Pt}^{2+}$ to $\mathrm{Pt}^{0}$ with a reasonable signal-concentration correlation (Fig. SI17-18). In conclusion, we have demonstrated here the feasibility of employing surface modified electrodes for the electrochemical adsorption and monitoring of Pt-based drugs from complex aqueous media. Our strategy is underpinned by the identification of commercially available TCEP as an efficient scavenger of Pt. Using UV-Vis and 1D ${ }^{31} \mathrm{P}-\mathrm{NMR}$ we have demonstrated that TCEP has a higher affinity 
for Pt than chelators found in nature, such as guanine or glutathione, or than representative thiol containing molecules. Moreover, these homogeneous-phase studies have allowed us to identify the nature, affinity and stoichiometry of the binding of TCEP to common Pt-based drugs such as cisplatin and carboplatin, a knowledge that should inform the future design of improved surfaces with highcapacity for the adsorption of Pt-based drugs. Immobilization of TCEP onto flat electrodes could be achieved through a simple EDC-mediated coupling onto a cysteamine-modified gold electrode. The interaction of Pt-based drugs with these TCEP-modified electrodes was evaluated using cyclicvoltammetry, impedance spectroscopy, X-ray photoelectron spectroscopy and contact angle, to demonstrate that a differential response could be observed for the TCEP-modified electrodes suggesting that Pt is preferentially absorbed on the TCEP sites and not on the pinholes at the gold surface. Appearance of a reductive current for the immobilization of $\mathrm{K}_{2} \mathrm{PtCl}_{4}$ on these TCEP-modified electrodes highlights the feasibility of using electrochemical filtration to monitor the concentration of Pt-based drugs in complex aqueous environments such as the blood stream. Altogether, these results demonstrate the potential of electrochemical devices for the development of new systems for the filtration and monitoring of Pt-based anticancer drugs. Our efforts to translate these results from flat surfaces into more applicable high-surface area systems with improved Pt adsorption, as well as the evaluation of other electrochemical techniques for the monitoring of Pt-based drugs in solution, will be reported in due course.

This article represents the first insight into the concept of the electrochemical hemofiltration of anticancer drugs. The implementation of this electrochemical platform in a real system will need however further studies including the effects of proteins and haemoglobin, as well the presence of other possible interference such as sugars.

\section{Methods}

\section{Electrode preparation}


Prior to any modification a gold electrode was flame-annealed and rinsed with ultrapure water (Elga, 18.2 $\mathrm{M} \Omega \mathrm{cm}, 1 \mathrm{ppb}$ total organic carbon). Subsequently, the electrode was immersed in a freshly prepared $100 \mathrm{mM}$ solution of cysteamine (Sigma Aldrich, $\geq 98 \%$ ) in water for 30 minutes. Afterwards, the cysteamine-modified electrode was rinsed with ultrapure water and immersed in a freshly prepared mixture (1:1 by volume) of solutions of $20 \mathrm{mM} \mathrm{TCEP} \cdot \mathrm{HCl}$ and $80 \mathrm{mM} \mathrm{EDC} \cdot \mathrm{HCl}$ for 60 minutes.

Stock solutions of TCEP. $\mathrm{HCl}($ Alfa Aesar, 98\%) and EDC. $\mathrm{HCl}$ (Fluorochem, >98\%) were made in 100 mM MES monohydrate (Alfa Aesar, 98\%) buffer and kept at $4{ }^{\circ} \mathrm{C}$ before its use. All the modification steps were performed at room temperature.

In the case of the high surface area gold electrodes used in XPS experiments, the gold electrodes were immersed in $250 \mathrm{mM}$ solution of cysteamine for 60 minutes in order to avoid the depletion of the reactants and ensure the ideal formation of the SAM.

The detailed description of the electrochemical measurements and the characterization by UV, XPS, NMR, EIS and contact angle are presented in the Supporting Information.

\section{Acknowledgments}

This work was supported by the University of Birmingham through PhD studentship (AK) and fellowships (PFT and PR), Wellcome Trust ISSF Grant 184ISSFPP (PFT) and John Evans Fellowship (PFT).

\section{Authors contribution}

PR, PFT and AK conceived the experiments and contributed to the experimental set-up and discussed the results. AK performed all experimental work. CSLD contributed in the NMR experiments and analysis. The XPS experiments and analysis of the data were performed by MW. AJK and AER contributed in the EIS and contact angle experiments. AER provided supervision and advice to AK whilst on secondment to the University of Southampton. All authors contributed to the 
analysis of the results, discussion, writing and revision of the manuscript. All authors have given approval to the final version of the manuscript.

\section{Conflicts of interest}

There are no conflicts to declare.

\section{Supporting Information.}

The Supporting Information is available free of charge on the ACS Publications website at

DOI: $x x x x x x$

The supporting information contains: complete experimental methods, UV-Visible

measurements, NMR spectra, XPS spectra, cyclic voltammetry and impedance

measurements and Contact angle measurements

\section{References}

(1) Rosenberg, B.; Van Camp, L.; Krigas, T. Inhibition of Cell Division in Escherichia coli by Electrolysis Products from a Platinum Electrode. Nature 1965, 205, 698.

(2) Rosenberg, B.; Vancamp, L.; Trosko, J. E.; Mansour, V. H. Platinum Compounds: a New Class of Potent Antitumour Agents. Nature 1969, 222, 385.

(3) Wong, E.; Giandomenico, C. M. Current Status of Platinum-Based Antitumor Drugs. Chemical Reviews 1999, 99, 2451-2466.

(4) Weiss, R. B.; Christian, M. C. New Cisplatin Analogues in Development. Drugs 1993, $46,360-377$.

(5) Kelland, L. The resurgence of platinum-based cancer chemotherapy. Nature Reviews Cancer 2007, 7, 573.

(6) Gately, D. P.; Howell, S. B. Cellular accumulation of the anticancer agent cisplatin: A review. British Journal Of Cancer 1993, 67, 1171.

(7) Von Hoff, D.; Schilsky, R.; Reichert, C.; Reddick, R.; Rozencweig, M.; Young, R.;

Muggia, F. Toxic effects of cis-dichlorodiammineplatinum (II) in man. Cancer treatment reports 1979, $63,1527$.

(8) Florea, A.-M.; Büsselberg, D. Cisplatin as an Anti-Tumor Drug: Cellular Mechanisms of Activity, Drug Resistance and Induced Side Effects. Cancers 2011, 3, 1351.

(9) Pingpank, J. F.; Libutti, S. K.; Chang, R.; Wood, B. J.; Neeman, Z.; Kam, A. W.; Figg, W. D.; Zhai, S.; Beresneva, T.; Seidel, G. D. Phase I study of hepatic arterial melphalan infusion and hepatic venous hemofiltration using percutaneously placed catheters in patients with unresectable hepatic malignancies. Journal of Clinical Oncology 2005, 23, 3465. 
(10) Yatzidis, H. A convenient haemoperfusion micro-apparatus over charcoal for the treatment of endogenous and exogenous intoxications. Its use as an effective artificial kidney, Procceding of the European Dialysis and Transplant Association,1964, 1, 83-87.

(11) Hagstam, K. E.; Larsson, L. E.; Thysell, H. Experimental studies on charcoal haemoperfusion in phenobarbital intoxication and uraemia, including histopathologic findings. Journal of Internal Medicine 1966, 180, 593-604.

(12) Webb, D. Charcoal haemoperfusion in drug intoxication. British journal of hospital medicine 1993, 49, 493-496.

(13) Graham, R. A.; Siddik, Z. H.; Hohn, D. C. Extracorporeal hemofiltration: a model for decreasing systemic drug exposure with intra-arterial chemotherapy. Cancer Chemotherapy and Pharmacology 1990, 26, 210.

(14) Chiavarino, B.; Crestoni, M. E.; Fornarini, S.; Scuderi, D.; Salpin, J.-Y. Interaction of Cisplatin with Adenine and Guanine: A Combined IRMPD, MS/MS, and Theoretical Study. Journal of the American Chemical Society 2013, 135, 1445-1455.

(15) Kasherman, Y.; Sturup, S.; Gibson, D. Is Glutathione the Major Cellular Target of Cisplatin? A Study of the Interactions of Cisplatin with Cancer Cell Extracts. Journal of Medicinal Chemistry 2009, 52, 4319-4328.

(16) Appleton, T. G.; Hall, J. R.; Ralph, S. F.; Thompson, C. S. M. Reactions of platinum(II) aqua complexes. 2. Platinum-195 NMR study of reactions between the tetraaquaplatinum(II) cation and chloride, hydroxide, perchlorate, nitrate, sulfate, phosphate, and acetate. Inorganic Chemistry 1984, 23, 3521-3525.

(17) Kolodziej, A.; Figueiredo, M. C.; Koper, M. T. M.; Fernandez-Trillo, F.; Rodriguez, P. Phosphate-mediated electrochemical adsorption of cisplatin on gold electrodes. Electrochimica Acta 2017, 248, 409-415.

(18) Nieboer, E.; Richardson, D. H. S. The replacement of the nondescript term "heavy metals' by a biologically and chemically significant classification of metal ions. Environmental Pollution Series B, Chemical and Physical 1980, 1, 3-26.

(19) Chen, S.; Jiang, H.; Wei, K.; Liu, Y. Tris-(2-carboxyethyl) phosphine significantly promotes the reaction of cisplatin with Sp1 zinc finger protein. Chemical Communications 2013, 49, $1226-1228$.

(20) Gregory, A. J.; Levason, W.; Noftle, R. E.; Le Penven, R.; Pletcher, D. Studies of platinum electroplating baths Part III. The electrochemistry of $\mathrm{Pt}(\mathrm{NH} 3) 4-\mathrm{x}(\mathrm{H} 2 \mathrm{O}) 2+2$ and $\mathrm{PtCl} 4-$ $\mathrm{x}(\mathrm{H} 2 \mathrm{O})(2-\mathrm{x})-\mathrm{x}$. Journal of Electroanalytical Chemistry 1995, 399, 105-113.

(21) Zhang, Y.; Guo, Z.; You, X.-Z. Hydrolysis Theory for Cisplatin and Its Analogues Based on Density Functional Studies. Journal of the American Chemical Society 2001, 123, 9378-9387.

(22) Chatt, J.; Duncanson, L. A.; Venanzi, L. M. Directing effects in inorganic substitution reactions. Part I. A hypothesis to explain the trans-effect. Journal of the Chemical Society (Resumed) $1955,4456-4460$.

(23) Hill, A. V. The possible effects of the aggregation of the molecules of haemoglobin on its dissociation curves. J. Physiol. 1910, 40, 4-7.

(24) Holt, M. S.; Nelson, J. H. Platinum (II) Complexes of (cyanoethyl)phosphines. Inorganic Chemistry 1986, 25, 1316-1320.

(25) Macdougall, J. J.; Nelson, J. H.; Mathey, F. Intramolecular and intermolecular equilibria and their pertinence to the mechanism of cis-trans isomerization of L2PtX2 complexes - 4coordinate and 5-coordinate platinum phosphole complexes. Inorganic Chemistry 1982, 21, 21452153.

(26) Alston, D. R.; Slawin, A. M. Z.; Stoddart, J. F.; Williams, D. J.; Zarzycki, R. Macrobicyclic Polyethers as Second Sphere Ligands for Tetraammineplatinum(II). Angewandte Chemie International Edition in English 1987, 26, 693-696.

(27) Raymo, F. M.; Stoddart, J. F. Second-Sphere Coordination. Chemische Berichte 1996, $129,981-990$. 
(28) Kolodziej, A.; Fernandez-Trillo, F.; Rodriguez, P. Determining the parameters governing the electrochemical stability of thiols and disulfides self-assembled monolayer on gold electrodes in physiological medium. Journal of Electroanalytical Chemistry 2018, 819, 51-57.

(29) Chow, E.; Hibbert, D. B.; Gooding, J. J. Voltammetric detection of cadmium ions at glutathione-modified gold electrodes. Analyst 2005, 130, 831-837.

(30) Seo, K.; Jeon, I. C.; Yoo, D. J. Electrochemical Characteristics of Ferrocenecarboxylate-Coupled Aminoundecylthiol Self-Assembled Monolayers. Langmuir 2004, 20, 4147-4154.

(31) Shin-ichiro, I.; Minehiko, I.; Daisuke, H.; Zhi Qiang, F.; Katsumi, N.; Takashi, K. Reductive desorption of carboxylic-acid-terminated alkanethiol monolayers from $\mathrm{Au}(111)$ surfaces. Journal of Electroanalytical Chemistry 1997, 428, 33-38.

(32) Boubour, E.; Lennox, R. B. Insulating Properties of Self-Assembled Monolayers Monitored by Impedance Spectroscopy. Langmuir 2000, 16, 4222-4228.

(33) Boubour, E.; Lennox, R. B. Potential-Induced Defects in n-Alkanethiol Self-Assembled Monolayers Monitored by Impedance Spectroscopy. The Journal of Physical Chemistry B 2000, 104, 9004-9010.

(34) Ozkaraoglu, E.; Tunc, I.; Suzer, S. X-ray induced reduction of Au and Pt ions on silicon substrates. Surface and Coatings Technology 2007, 201, 8202-8204.

(35) Karadas, F.; Ertas, G.; Ozkaraoglu, E.; Suzer, S. X-ray-Induced Production of Gold Nanoparticles on a SiO2/Si System and in a Poly(methyl methacrylate) Matrix. Langmuir 2005, 21, 437-442.

(36) Scharifker, B.; Hills, G. Theoretical and experimental studies of multiple nucleation. Electrochimica Acta 1983, 28, 879-889.

(37) Scharifker, B. R.; Mostany, J.; Palomar-Pardave, M.; Gonzalez, I. On the theory of the potentiostatic current transient for diffusion-controlled three-dimensional electrocrystallization processes. Journal of the Electrochemical Society 1999, 146, 1005-1012.

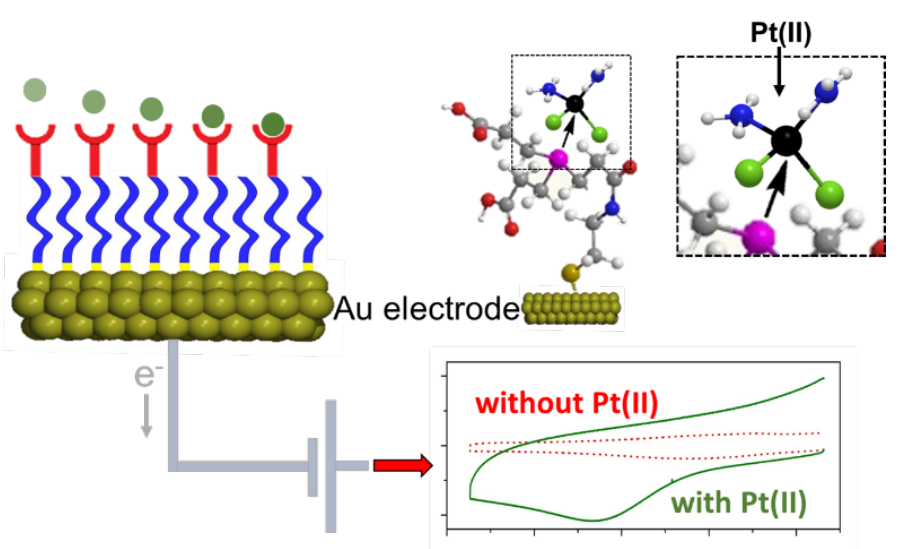

